\title{
The effect of olivine content and curing time on the strength of treated soil in presence of potassium hydroxide
}

\begin{abstract}
When olivine (Mg2SiO4) is activated with potassium hydroxide $(\mathrm{KOH})$, it acquires the ability to improve the unconfined compressive strength of soil. This paper investigates the use of olivine for soil stabilisation through alkaline activation by focusing on the role of different alkali activated olivine contents (5-20 wt\%) in stabilising native soil at different curing durations. The strength results were supported by a detailed microstructural and compositional analysis including scanning electron microscopy, energy-dispersive X-ray spectroscopy and X-ray diffraction. Use of olivine in the presence of $\mathrm{KOH}$ increased the shear strength of soil up to 7.4 MPa in 90 days as a result of the formation of brucite, quartz and mullite in the structure of treated soil. This achievement implies a tremendous effect of olivine on the strength behaviour of treated soil. These results provide essential information which is significant from an environmental perspective as it offers a low energy alternative to existing technologies, for soil stabilisation.
\end{abstract}

Keyword: Olivine; Alkaline activation; Soil stabilization; Microstructure analysis 